\title{
Structuring the Quest for Strategic Alignment of Artificial Intelligence (AI): A Taxonomy of the Organizational Business Value of AI Use Cases
}

\author{
Christian Engel \\ University of St. Gallen \\ christian.engel@unisg.ch
}

\author{
Julius Schulze Buschhoff \\ University of St. Gallen \\ juliusconstantin.schulzebuschhoff@, \\ student.unisg.ch
}

\author{
Philipp Ebel \\ University of St. Gallen \\ philipp.ebel@unisg.ch
}

\begin{abstract}
The deployment of Artificial Intelligence (AI) in businesses is said to provide significant benefits to organizations. However, many businesses struggle to align single AI use cases with the overall strategic business value contribution. Thus, we investigate the strategic characteristics that determine the business value contribution of AI use cases at an organizational level. We draw on academic literature and 106 AI use cases to develop a conceptually sound and empirically grounded taxonomy of the organizational business value of AI use cases. With the developed taxonomy, decision-makers are presented with a tool to systematically align AI use cases with strategic objectives. Moreover, our findings reveal how an AI use case can generate different business value contributions in different contexts, which provides researchers with a conceptual frame for informing their empirical research endeavors at the organizational level.
\end{abstract}

\section{Introduction}

The continuous improvements of Artificial Intelligence (AI) offer a proficient way to transform and innovate organizational processes [46]. The fact that many companies have already understood the potential benefits of AI can be clearly seen in the increase in investments in AI technologies, which are expected to increase from $\$ 12$ billion in 2017 to $\$ 15$ trillion by the end of 2030 across all industries [10, 42]. Researchers generally agree that AI can increase a companies' ability to innovate and at the same time fosters their productivity, which ultimately enhances their competitive advantage [28]. However, with the rise of AI, organizations also face a potentially fundamentally changing competitive landscape, and a reshape of entire industries [1]. This implies that novel business models will arise [17], causing entire value chains to be redesigned while putting businesses into new competition with each other [15]. At the same time, many companies face considerable difficulties when implementing AI in their organizations. "More than
$60 \%$ of [companies] say that a strategy for $\mathrm{AI}$ is urgent for their organizations, but only half of those say their organizations have a strategy in place" [42, p.12]. This issue is affirmed by other researchers as well [e.g., 1], who state that there is often a lack of strategic understanding of where AI can be used in the organization and for what strategic purpose AI needs to be aligned. This lack of strategic alignment leads to synergy effects among existing or new AI use cases being lost [1]. Here, the specific challenge is to identify existing or design novel AI use cases that fit the business's needs of an organization and generate the desired business value contribution. After all, an inadequately planned application of an AI use case can not only fail to generate value, but even lead to value destruction, as illustrated by examples, such as Amazon's gender-biased AI resume screener [35].

To avoid these pitfalls and to support organizations in realizing business value from AI, we follow the calls for research on supporting organizational decisionmakers in assessing AI use cases in terms of their anticipated strategic business value contribution [e.g., 3, 16]. Therefore, this paper pursues the goal to contribute to the organizational ability of better assessing AI use cases in regard to their business value contribution from a strategical perspective. To achieve this goal, we pose the following research question: What are the conceptually sound and empirically grounded characteristics that determine the business value contribution of AI use cases at the organizational level?

To answer this research question, we develop a taxonomy of the organizational business value of AI use cases according to the method proposed by Nickerson et al. [34]. Accordingly, the remainder of this paper is structured as follows: First, we provide a brief overview of the conceptual foundations and related work before we describe the taxonomy development approach and present the final taxonomy as well as its evaluation and application. Subsequently, we discuss our findings and present some final concluding remarks. To practitioners, the taxonomy provides a tool for assessing and identifying the value of and the area for implementation 
of AI use cases in one's own business. It supports an informed decision-making process for an organization's digital transformation and thus helps to remain competitive in the market by taking advantage of AI. From a research perspective, the taxonomy can be used to analyze how the same AI use case can generate different business values in different contexts.

\section{Foundations of AI and business value}

Each research discipline has a slightly different understanding of AI [3]. While, from a more epistemological viewpoint, AI refers to the study of knowledge acquisition and representation in a logicoriented propositional formalism and the manipulation of this knowledge with logical operations [37], computer scientists focus on the machine's ability to mimic human behavior and hence human intelligence by logical deduction [26]. This goes along with solving complex tasks, and the adaptability of AI to the environment it is operating in. In this paper, we refer to the latter notion of AI presented above.

Before investigating the topic of AI benefits further, it is important to define the term business value first, as there is still no consensus definition present [36]. Some scholars refer to it as "value", "benefit", "outcome", or "worth" [44], while others describe it as "economic impact" [23], or "organizational performance" [29]. Schryen [43] illustrates further that for the assessment of business value, scholars examine different parameters, which can be divided into financial measures (relationships between information systems investment and productivity, return on sales) and nonfinancial measures (organizational capabilities, strategic position). For the purpose of this paper, we adopt the definition by Schryen [44, p.141]: "IS business value is the impact of investments in particular IS assets on the multidimensional performance and capabilities of economic entities at [an organizational level], complemented by the ultimate meaning of performance in the economic environment". This notion of business value has also been adopted by other researchers from the realm of organizational AI research and shown to be a useful research lens [e.g., 2, 9, 31, 32, 47]. Besides presenting a definition of business value, Schryen [43] propose a model of business value. It outlines four factors impacting value creation through IS investments: "context/environmental factors", "IS investments" themselves, "non-IS investments", and "lag effects" [44, p.144]. When presenting this model, Schryen points out that "these subfields have not explained sufficiently how, why and when IS investments create business value," thus recommending further examination of the "grey box of [...] IS business value creation" [44, p.144].

\section{Related work}

The topic of AI is considered to be one of the most significant drivers of economic growth over the next couple of years [46]. Through the large amounts of data created by all kinds of sensors and objects, companies are exposed to opportunities to fundamentally improve their business practices across all departments along the entire value chain [46]. Bughin et al. [7] elaborate on this by giving detailed examples of how certain departments may benefit, summarizing these by categorizing them into projection (better predicting trends and demands), optimizing (sourcing and evaluating prototypes), production (improving products and services), promotion (price discrimination and targeting customers, and provision (increasing customer satisfaction by offering fuller and more customized services). Other studies examine the impacts of AI on the economy [1], highlighting the new competitive situation that businesses are exposed to, as well as the challenges faced when trying to derive value from AI [2]. Here, Alhosni et al. [2] identify the six challenges as follows: (1) alignment of AI case to strategy, (2) relative benefits to realize, (3) top management support, (4) AI talent needed, (5) effective use of data, and (6) AI compatibility. As this selection of studies demonstrates, scholars have performed extensive research on the impact AI can have on organizations, and the prerequisites necessary for its implementation. Nevertheless, this still leaves many companies wondering how to strategically approach this subject to find and assess whether and how an AI use case actually enhances business value [e.g., 2, 3, 7]. Hence, many studies stress the importance of a more detailed investigation of AI use case deployment with great focus on guidelines for strategically examining the potential business value of $\mathrm{AI}$, to allow organizations to benefit from the advantages of AI [e.g., 3, 7, 9, 19].

To counteract this, numerous researchers have already published taxonomies to engage in analysis of AI-related topics. Fadler and Legner [14] built a taxonomy to explore the influence of AI on enterprise data management. Dealing with the struggle of unstructured knowledge management (UKM), Cheung et al. [8] built a taxonomy to offer a solution to better extract relevant information from the large databases needed for AI. Dellermann et al. [12] built a taxonomy to analyze the different forms of collaboration between human and machine, especially focusing on the distribution of tasks and the technology used for this. Wilson and Daugherty [48] examined "collaborative intelligence" between human and machines, indicating in a taxonomy, in which areas value can be created, accompanied by illustrative examples. Hartmann et al. [19] developed a taxonomy that indicates several 
dimensions to capture value from big data - focusing predominantly on start-ups. Yampolskiy [49] tackles the topic of disadvantages that arise with AI technology by introducing a taxonomy to investigate the potential danger of AI. Similarly, Kukačka et al. [24] propose solutions to prevent AI from having negative impacts on society. Moreover, Amer and Maul [4, p.29] sought out ways to scale AI technology, shifting the focus toward analysis of "properties and relationships of different variants" in regard to neural networks, thus finally providing a taxonomy to "systematically implement neural modularity". Taking on a broader context, Hunke et al. [20] adopted Nickerson et al.'s [34] taxonomy development approach by analyzing the anatomy of analytics-based services to develop a taxonomy that indicates important dimensions for service design. However, these studies do not offer a structure for analyzing the fundamental strategic characteristics of how AI technology (respectively AI use cases) contributes to business value creation at the organizational level. Thus, we develop a taxonomy outlining the core levers of AI use cases that are relevant for strategic decision-making on AI in organizations.

\section{Method}

We follow Nickerson et al.'s [34] taxonomy development method to study AI business value in organizations. Nickerson et al.'s [34] method constitutes an iterative approach. It offers researchers the opportunity to both conceptually, on a literature basis, and empirically, based on instantiations observable in the real world, construct taxonomies. Their method has two preliminary instructions that need to be pursued before actually starting the taxonomy development process. First, the we need to define a metacharacteristic, which is the driving lens of the taxonomy and which all taxonomy dimensions and characteristics logically follow. Secondly, we need to define ending conditions for the taxonomy development process to terminate. We adapted the objective and subjective ending conditions from Nickerson et al. [34], also displayed in Table 4.

We use the following notation [34]: $\mathrm{T}=\left\{\mathrm{D}_{i}, i=1, \ldots\right.$, $\left.n \mid \mathrm{D}_{i}=\left\{\mathrm{C}_{i j}, j=1, \ldots, k_{i} ; k_{i \geq 2}\right\}\right\}$. Taxonomy $\mathrm{T}$ consists of a number of dimensions $\mathrm{D}_{i}(i=1, \ldots, n)$, which in turn are true for $k_{i}\left(k_{i} \geq 2\right)$ mutually exclusive and collectively exhaustive characteristics $\mathrm{C}_{i j}\left(j=1, \ldots, k_{i}\right)$.

There are two modules of the taxonomy building process that can be applied iteratively. First, the conceptual-to-empirical module focuses on conceptualizing the dimensions of the taxonomy without examining real-world instantiations. Second, the empirical-to-conceptual module focuses on deriving dimensions and characteristics based on real-world instantiations. Thus, in the second module of the method we need to identify a subset of existing real-world instantiations to analyze them with regard to their mutual characteristics. Then, we group the identified characteristics into dimensions to develop a (revised) version of the taxonomy. After each iteration, we assess if the ending conditions according to Nickerson et al. [34] are fulfilled. If this is not the case, we select one of the method's modules again to start a new iteration.

The taxonomy must be evaluated to assess its usefulness and value for practitioners and researchers [34]. Apart from meeting the ending conditions, which is a precondition for finishing the process of taxonomy development, the taxonomy's usefulness can be measured by letting other researchers use and evaluate it [34]. We decided to engage in both qualitative and quantitative evaluation. For the qualitative evaluation, we conducted two semi-structured interviews [25] with AI experts. These expert interviews were held at the point when we regarded the taxonomy as needed to be challenged by potential users for further maturity. For the quantitative evaluation, we let four AI experts collate 10 randomly selected AI use cases into the taxonomy to determine an intercoder agreement. This goes hand in hand with the approach of Nahm et al. [33].

\section{Results}

Here, we outline the development iterations performed. Subsequently, we present the final taxonomy of the organizational business value of AI use cases, followed by the taxonomy's evaluation. Finally, we showcase the application of the taxonomy. Table 1 provides an overview of the iterations that we conducted when developing the taxonomy and the respective ending conditions.

\section{Table 1. Summary of ending conditions}

\begin{tabular}{|l|c|c|c|c|c|}
\hline \multicolumn{1}{|c|}{ Ending Conditions/Iteration (i-v) } & i & ii & iii & iv & v \\
\hline 1) All objects or a representative sample examined & & & & & $\bullet$ \\
\hline 2) No object merged or split in last iteration & $\bullet$ & $\bullet$ & $\bullet$ & $\bullet$ & $\bullet$ \\
\hline $\begin{array}{l}\text { 3) At least one object classified under every } \\
\text { characteristic of every dimension }\end{array}$ & & $\bullet$ & $\bullet$ & $\bullet$ & $\bullet$ \\
\hline $\begin{array}{l}\text { 4) No new dimension or characteristic added in } \\
\text { last iteration }\end{array}$ & & & $\bullet$ & & $\bullet$ \\
\hline $\begin{array}{l}\text { 5) No dimensions or characteristics merged or split } \\
\text { in last iteration }\end{array}$ & $\bullet$ & & & & $\bullet$ \\
\hline 6) Every dimension is uniqu and not repeated & $\bullet$ & & $\bullet$ & $\bullet$ & $\bullet$ \\
\hline $\begin{array}{l}\text { 7) Every characteristic is unique within its } \\
\text { dimension }\end{array}$ & & & $\bullet$ & $\bullet$ & $\bullet$ \\
\hline 8) Each cell is unique and not repeated & & $\bullet$ & $\bullet$ & $\bullet$ & $\bullet$ \\
\hline 9) Conciseness & & & & $\bullet$ & $\bullet$ \\
\hline 10) Robustness & & $\bullet$ & & $\bullet$ & $\bullet$ \\
\hline 11) Comprehensiveness & & & & $\bullet$ & $\bullet$ \\
\hline 12) Extendibility & $\bullet$ & $\bullet$ & $\bullet$ & $\bullet$ & $\bullet$ \\
\hline 13) Explanatory Character & & & & $\bullet$ & $\bullet$ \\
\hline
\end{tabular}


taxonomy were implemented. For instance, we merged dimensions D4 "Sources of Value Creation" and D6 "AI Business Functionalities", as these characteristics described the same phenomenon but from different perspectives. As a result, the newly formed dimension D4 was named "Sources of Business Value Improvement". Merging the dimensions led to three new characteristics. There was also a merger within dimension D3 "Market Oriented Investment", as the characteristic "Research Investment" was merged with the characteristic "Competitive Edge Investment", followed by a split through which the characteristic "Performance Excellence Investment" was created. Furthermore, in dimension D7 "Benefit to Business Value", several characteristics were merged for reasons of comprehensiveness. In addition, dimension D2, formerly called "AI Use Case Alignment to Overall Strategy", was renamed "Time Oriented Strategic Alignment". This was intended to make it clearer that this dimension focused on what time-oriented vision the AI use case contributed to. Moreover, dimension D3

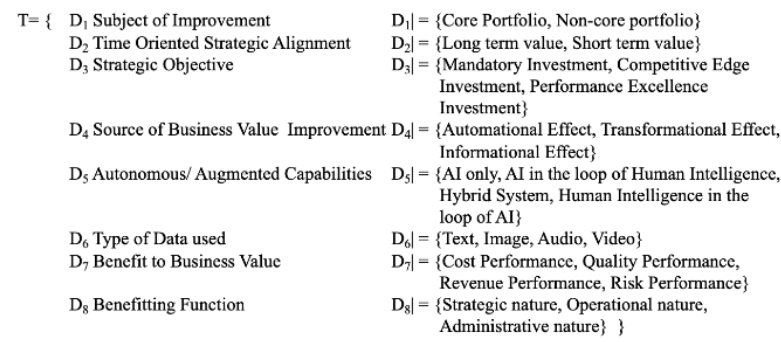

Figure 3. Taxonomy after third iteration

was renamed "Strategic Objective". This new description was intended to more strongly emphasize the dimension's focus on the goal that is targeted when applying the AI use case. Finally, dimension D8 "Benefiting Department" was renamed to "Benefiting Function" to shift the focus single departments to a higher, less granular perspective, indicating the context, in which the AI use case creates value. Accordingly, the characteristics in this dimension were changed.

Iteration 4: As not all objective and subjective ending conditions were met (see Table 1), we addressed the empirical module again. However, instead of drawing on the database, we conducted two AI expert interviews. We selected two interview partners who are experts in the field of AI one from the tech industry (six years of AI experience) and one from consulting (five

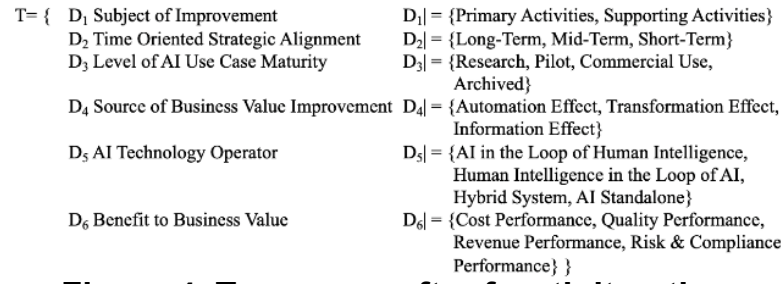

Figure 4. Taxonomy after fourth iteration years of AI experience). The interviews were conducted anonymously in succession. Based on the experts' feedback, valuable improvements could be undertaken. However, some objective ending conditions were no longer fulfilled. Condition 1 was the main driver calling for a fifth iteration, as not all AI use cases of the previously established data set had been examined yet.

Iteration 5: For the fifth iteration, the empirical approach was used again. Here, we examined the final 16 AI use cases of the accumulated data set. The intention behind this was to apply a final check to the modified taxonomy after integrating the experts' feedback. As no changes to the construction of the taxonomy were made, all ending conditions were met (see Table 1). Hence, the development process was concluded.

\subsection{Taxonomy of organizational business value of AI use cases}

The final taxonomy entails six dimensions with respective characteristics, which will be explained here. Table 4 displays the taxonomy's body.

For some dimensions, we allow the characteristics to be non-exclusive because as one use case can contribute to more than one characteristic when creating business value at the organizational level. This is in line with other scholars [e.g., 21]. Thus, we include the abbreviations "N" (non-exclusive) and "E" (exclusive) in each dimension in Table 4.

The dimension "Subject of Improvement" outlines which business area is targeted for improvement by the AI use case. This classification is essential to determine whether the AI use case is capable of deriving additional business value in the area where the business challenge is located [6]. Possible areas are summarized in the two characteristics "Primary Activities" and "Supporting

\section{Table 4. Taxonomy of organizational business} value of Al use cases

\begin{tabular}{|c|c|c|c|c|c|}
\hline Dimension & \multicolumn{4}{|c|}{ Characteristics } & $E / N$ \\
\hline $\begin{array}{c}\text { Subject of } \\
\text { Improvement }\end{array}$ & \multicolumn{2}{|c|}{ Primary Activities } & \multicolumn{2}{|c|}{ Supporting Activities } & E \\
\hline $\begin{array}{c}\text { Time Oriented } \\
\text { Strategic Aliggment } \\
\end{array}$ & Long-Term & \multicolumn{2}{|c|}{ Mid-Term } & Short-Term & E \\
\hline \begin{tabular}{|c|} 
Level of AI Use Case \\
Maturity
\end{tabular} & Research & Pilot & Commercial Use & Archived & E \\
\hline $\begin{array}{l}\text { Source of Business } \\
\text { Value Improvement }\end{array}$ & Automation Effect & \multicolumn{2}{|c|}{ Transformation Effect } & Information Effect & $\mathrm{E}$ \\
\hline $\begin{array}{c}\text { AI Technology } \\
\text { Operator }\end{array}$ & $\begin{array}{c}\mathrm{AI} \text { in the Loop } \\
\text { of Human } \\
\text { Intelligence }\end{array}$ & $\begin{array}{l}\text { Human } \\
\text { Intelligence in } \\
\text { the Loop of AI }\end{array}$ & Hybrid System & AI Standalone & E \\
\hline $\begin{array}{c}\text { Benefit to Business } \\
\text { Value }\end{array}$ & $\begin{array}{c}\text { Cost } \\
\text { Performance }\end{array}$ & $\begin{array}{c}\text { Quality } \\
\text { Performance }\end{array}$ & $\begin{array}{l}\text { Revenue } \\
\text { Performance }\end{array}$ & $\begin{array}{c}\text { Risk \& } \\
\text { Compliance } \\
\text { Performance }\end{array}$ & $\mathrm{N}$ \\
\hline
\end{tabular}

Activities", for which the we refer to Porter's value chain [38], as well as Wamba-Taguimdje et al.'s research model [46]. The characteristic "Primary Activities" includes all activities that are associated directly or indirectly with enhancing the organization's value proposition [39]: Direct activities are activities 
that are actively included in deriving additional business value in utilizing AI to either add or improve features of existing products and services or to design completely new products and services. Meanwhile, indirect activities are associated with supporting these direct activities. Finally, there are activities guaranteeing the quality of the primary activities, which include, among others, "monitoring, inspecting, testing, reviewing, checking, adjusting and reworking" [40, p.56]. The characteristic "Supporting Activities" on the other hand describes all activities within the business that are carried out around the primary activities to support them and keep the business running [39]. These activities occupy the fields of firm infrastructure, human resource management, technology development, and procurement [39]. Again, they include direct activities (activities that are labeled as supporting activities), indirect activities (assisting supporting activities) and quality-ensuring activities (maintaining the quality of supporting activities [39]. Based on the AI use cases investigated over the course of this paper, we concluded that an AI use case will have most impact on business value contribution if only one of the two characteristics is the focus of the AI use case, thus making this dimension exclusive ("E").

"Time Oriented Strategic Alignment" describes the time horizon-oriented goal to which the AI use case is supposed to contribute. As stressed by Coombs et al. [9] and Mikalef and Gupta [32], clearly indicating whether the AI use case focuses on broad organizational priorities or isolated business problems is highly relevant for the expected business value contribution(s). We differentiate here between "Long-Term", "MidTerm", and "Short-Term" time spans. As stressed by Mikalef and Gupta [32], the "Short-Term" timespans are mostly found with AI use cases in the context of isolated business problems. Here, the AI use case is expected to derive "instant" improvements in regard to the current issue. On the other hand, AI use cases that fall under the "Mid-Term" characteristic have a broader perspective. They focus on more than just urgent isolated challenges, but do not have the means to contribute to the long-term future orientation of the organization. In contrast, "Long-Term” AI use cases are aligned to the organization's long-term strategy. These AI use cases help achieve the organization's vision.

The dimension "Level of AI Use Case Maturity" focuses on the use case's level of development, thus indicating how technically developed and applicable the AI use case is in the real world. The dimension and associated characteristics were formed based on insights from Mankins [27], who investigated the phenomenon of technology readiness levels. This dimension provides practitioners with valuable information about the feasibility of the AI use cases. The characteristics of this dimension are the following: "Research", "Pilot", "Commercial Use", and "Archived". The "Research" stage includes the phases of researching and planning the AI technology behind the AI use case. At the "Pilot" stage, the concept of the AI use case is tested in a feasibility study to see if the anticipated business value contribution is likely to be realized. At the "Commercial Use" stage, the AI use case is already deployed in practice to create business value. The last characteristic is "Archived", which indicates that the AI use case is not being commercially used at the moment. This can be due to numerous reasons, such as low user acceptance, liability issues that cause the anticipated advantages of the AI use case to be outweighed by the disadvantages, no suitable real-world problem application found or an insufficiently developed AI technology.

The "Source of Business Value Improvement" dimension addresses the kind, in which the AI use case will generate business value in order to contribute to the time horizon-oriented goal. Here, the characteristics are the "Automation Effect", the "Transformation Effect", and the "Information Effect", according to WambaTaguimdje et al. [47, p.1902]. The "Automation Effect" will automate processes within the organization with a great emphasis on repetitive manual tasks. This will allow the business to leverage rationalization potential. The "Transformation Effect" is used to foster innovation by supporting design and creativity (Mikalef et al., 2019). This refers to products and services becoming smart, thus making the benefit of the product the basis of the value proposition for the customer. The "Information Effect" uses data for improved decisionmaking for both internal aspects within the organization and for the external environment. With the help of decision support, internal aspects within the company can be improved [45], predominantly focusing on (operational) processes. The data used for this is mainly generated by the organization itself. In regard to external decision-making, the customer or user is in focus. Here, the information effect derives insights about the customer or end user by using data generated by the external environment (mainly by customers or end users themselves), with the goal of providing individual useradapted offerings. This allows for an offering that is oriented around the customers or users, thus strengthening their satisfaction.

The "AI Technology Operator" dimension indicates the operator of the applied AI use case, which can either be the AI technology, a human, or both entities. Even though, this dimension is quite technical, it makes a significant contribution to the strategic analysis of an AI use case. The identification of the operator behind the AI use case allows for further assessment of the corresponding implications, such as the degree of influence on the business value 
contribution the company has itself (i.e., the more tasks of the AI use case are assigned to the AI, the lower is the human influence), or how much increase in workload will come along with it (the more tasks of the AI use case are assigned to human workers, the higher will be the workload). As scholars state, AI is used to augment human capabilities [5], while "humans can still offer a more holistic, intuitive approach" [23, p.577], focusing on more creative assignments [21]. We refer to Davenport and Ronanki [11], Ransbotham et al. [40], as well as Dellermann et al. [13] for the different forms of AI collaboration with human intelligence. The first characteristic is "AI in the Loop of Human Intelligence”, which describes AI performing easy-toautomate tasks or providing decision support to humans $[13$, p.639]. Second, there is "Human Intelligence in the Loop of AI", which labels the process of AI working under strong human supervision [13, p.639]. The AI is not yet advanced enough to solve problems alone, as humans need to assist in terms of the "generation of algorithms, [...] training or debugging models [or] making sense of unsupervised approaches such as data clustering" [13, p.639]. In addition to these two characteristics, there is also the "Hybrid System". The Hybrid System is based on the concept of hybrid intelligence, where the AI and the human are considered to be equal partners [13, p.639], as complicated tasks are approached using the strength of both parties. Schwartz et al. [44], who describe those constructs with the term hybrid teams and investigate different structures of these, claim that due to the heterogeneity of the team members, the expected results are greater than those of either party solving the problem individually. Jain et al. $[22$, p.250] refer to such projects as "human computational problem[s]," as these are often problems that "cannot be solved by either computer or humans alone". The final characteristic in this dimension is " $A I$ Standalone". This characteristic describes an AI working autonomously, so that no human interaction is required. For reasons of completeness, it is necessary to state that a fifth characteristic, "Human Standalone", could also be added. Here, only human workforce is deployed. However, as this would be far off-topic from the AI subject of this paper and does not contribute to an understanding of business value contribution of AI technology, we decided to exclude this characteristic.

The "Benefit to Business Value" dimension describes the direct benefit(s) that can be realized by applying the AI use case, and thus the specific business value that is being targeted. This possibly encompasses the goal of improving internal or external processes, following new regulations, increasing quality, or improving productivity, which all lead directly or indirectly to an enhanced competitive advantage [2]. While analyzing the collected data set, we found that from a broader perspective, in most cases most of the characteristics will apply, if examined from a long-term perspective. Hence, when identifying the potential benefit(s), it is important to examine only the direct and not the indirect benefit(s), as otherwise the quality of this dimension is diluted. The dimension's characteristics are thus the following: "Cost Performance", "Quality Performance", "Revenue Performance", and "Risk and Compliance Performance". "Cost Performance" summarizes two categories: on the one hand, actual measures to reduce costs, and on the other hand, actions that improve operational performance, in turn making the organization more efficient [48]. "Quality Performance" includes aspects that enhance the quality of products and services. "Revenue Performance" focuses on the customer by improving the way customers are for instance segmented or targeted, or the way prices are set [17]. Hence, it aims to increase the revenue. Finally, "Risk and Compliance Performance" encompasses actions taken to reduce the liability of an organization. This also includes in a broader interpretation the reduction of unethical behavior. Furthermore, it also focuses on prohibiting illegal action and the compliance to new laws and regulations.

\subsection{Evaluation}

To evaluate the usefulness of the taxonomy we calculated the intercoder agreement in accordance with Nahm et al. [33] by letting four participants collate 10 randomly chosen $\mathrm{AI}$ use cases from the data set into the taxonomy. The participants had not seen the taxonomy before. However, all had a professional business background, ensuring that they would understand the terms used in the taxonomy. The inter-coder agreement was calculated to test the taxonomy's "reliability and construct validity" $[34$, p.1]. In Table 5 the dimensionspecific hit ratios are listed.

\section{Table 5. Evaluation results}

\begin{tabular}{lc}
\multicolumn{1}{c}{ Dimension } & Hit Ratio (\%) \\
\hline $\mathrm{D}_{1}$ Subject of Improvement & 85 \\
$\mathrm{D}_{2}$ Time Oriented Strategic Alignment of AI Use Case & 77 \\
$\mathrm{D}_{3}$ Level of AI Use Case Maturity & 100 \\
$\mathrm{D}_{4}$ Sources of Business Value Improvement & 83 \\
$\mathrm{D}_{5}$ AI Technology Operator & 85 \\
$\mathrm{D}_{6}$ Benefit to Business Value & 81
\end{tabular}

Overall, it can be claim that though there are slight outliers, the dimension-specific hit ratios overall can be regarded to be at a high level ( $85 \%$ on average), indicating good agreement between the participants.

\subsection{Application}

In addition to the intercoder agreement, we showcase the application of the taxonomy in one 
exemplary use case, which was retrieved from the data set used for taxonomy development. This shall show its usefulness for strategic assessment of AI use cases.

The focal AI use case revolves around accelerating Table 6: Classification of "Danske Bank" case

\begin{tabular}{|c|c|c|c|c|c|}
\hline Dimension & \multicolumn{4}{|c|}{ Characteristics } & $E / \mathbf{N}$ \\
\hline $\begin{array}{l}\text { Subject of } \\
\text { Improvement }\end{array}$ & \multicolumn{2}{|c|}{ Primary Activities } & \multicolumn{2}{|c|}{ Supporting Activities } & E \\
\hline \begin{tabular}{c|} 
Time Oriented \\
Strategic Alignment
\end{tabular} & Long-Term & \multicolumn{2}{|c|}{ Mid-Term } & Short-Term & E \\
\hline $\begin{array}{l}\text { Level of AI Use Case } \\
\text { Maturity }\end{array}$ & Research & Pilot & Commercial Use & Archived & E \\
\hline $\begin{array}{l}\text { Source of Business } \\
\text { Value Improvement }\end{array}$ & Automation Effect & \multicolumn{2}{|c|}{ Transformation Effect } & Information Effect & E \\
\hline $\begin{array}{l}\text { AI Technology } \\
\text { Operator }\end{array}$ & $\begin{array}{l}\text { AI in the Loop } \\
\text { of Human } \\
\text { Intelligence }\end{array}$ & $\begin{array}{l}\text { Human } \\
\text { Intelligence in } \\
\text { the Loop of AI }\end{array}$ & Hybrid System & AI Standalone & E \\
\hline $\begin{array}{l}\text { Benefit to Business } \\
\text { Value }\end{array}$ & $\begin{array}{c}\text { Cost } \\
\text { Performance }\end{array}$ & $\begin{array}{c}\text { Quality } \\
\text { Performance }\end{array}$ & $\begin{array}{l}\text { Revenue } \\
\text { Performance }\end{array}$ & $\begin{array}{c}\text { Risk \& } \\
\text { Compliance } \\
\text { Performance }\end{array}$ & $\mathrm{N}$ \\
\hline
\end{tabular}

identity verification for new and existing customers at Danske Bank [16]: In this case, Danske Bank aimed to increase customer satisfaction by introducing an AI system provided by the company Behaviosec to its online services. The AI was designed to track fraudsters who hacked into Danske Bank customers' online accounts. Instead of only identifying the customer in the beginning of the online banking process, the AI system tracks the customers the whole time they are logged in. By doing so, it can compare the current customer behavior to the behavior the algorithm would expect.

Explanation of classification (see Table 6): Danske Bank provides payment, credit, and capital transaction services in exchange for payment as its core portfolio. Thus, online banking is part of Danske Bank's portfolio, which implies that ensuring non-fraudulent activity in online banking indirectly supports its primary activities. Thus, for D1 "Subject of Improvement", the characteristic "Primary Activity" is selected. In regard to D2 "Time Oriented Strategic Alignment", "MidTerm" applies to this AI use case, because with this AI technology, the bank does not solve a single business problem, but applies a possible solution to the issue of online fraud, which is and will remain highly relevant. However, as in the long run it is highly likely that the way (online) banking is performed will differ from the way it is conducted today, these security measures will not be sufficient enough to prevent fraudulent behavior in the long-run. The AI use case is currently still being tested. Hence, for D3 "Level of AI Use Case Maturity" the "Pilot" stage is selected. For D4 "Source of Business Value Improvement", the "Information Effect" applies, as data generated by the user is analyzed in order to determine whether the user is legitimate or fraudulent. Moreover, to determine the characteristic of D5 “AI Technology Operator”, the AI technology must be more closely investigated. Behaviosec explains that the algorithm works alone when tracking and analyzing the user's behavior and alerts if fraud is detected. However, the next steps - going after the fraudster and stopping all financial actions and requests made in this context - are the responsibility of the relevant bank representative. In other words, the AI gives a statement for each user upon which the bank representative must act. Therefore, the characteristic "AI in the Loop of Human Intelligence" is assigned. Coming to the benefits, which the AI use case should provide at the organizational level, two characteristics in D6 "Benefit to Business Value" can be identified: First, as the AI technology is able to reduce fraud, "Risk and Compliance Performance" applies; the risk of customers becoming victims of criminal activity is reduced. Second, this increases customer satisfaction. Thus, customers will engage more in online banking activities, which can lead to an increase in Danske Bank revenue. Hence, "Revenue Performance" also applies.

\section{Discussion}

The developed taxonomy shall contribute to closing the research gap of strategically assessing AI use cases in terms of their potential business value contribution at the organizational level. As presented in the related work section of this paper, extant studies do not offer a structure for analyzing the fundamental strategic characteristics of how AI technology (respectively AI use cases) contributes to business value creation at the organizational level. Thus, we conceptually and empirically identified the core levers of AI use cases that are relevant for strategic decision-making on AI in organizations. The structure of the taxonomy shall make the complexity of strategic analysis of AI use cases more manageable without excluding relevant information. The main beneficiaries are AI decision-makers. In fact, the taxonomy provides them with a tool to classify, examine, and compare different AI use cases prior to implementation in their business. Thus, the taxonomy supports the process of informed organizational decision-making, helping organizations to take advantage of AI's benefits. In addition, especially for practitioners who are not yet very familiar with the concept of AI, the taxonomy's structure allows for the prioritization of an area within the business, in which an AI use case should create business value. By analyzing AI use cases from a strategic perspective with respect to business value contribution at the organizational level, researchers can further deepen their understanding of the factors affecting the organizational business value of AI use cases. Furthermore, the taxonomy provides researchers with a conceptual frame for informing their empirical research endeavors at the organizational level. With the insights gained through the taxonomy how one AI use case can create different forms of business value in different organizational contexts, a more in-depth theorization of AI in organizations shall be enabled. 


\section{Limitations}

Of course, this research does not come without limitations. First, the generalizability of the results found is limited, as it only proves the taxonomy's coherency in categorizing known AI use cases and we used only one case of Danske Bank to showcase the applicability of the taxonomy. Second, the taxonomy's level of analysis is from a highly strategic perspective. Therefore, the taxonomy does not take into consideration aspects such as the necessary resources including human AI experts, necessary infrastructure, and data sets - nor does it highlight the time it takes to implement the AI use case. Thirdly, as one AI use case can generate various forms of business value in different settings, it might be possible that there are further factors supporting this than the taxonomy was able to identify, which however is in line with the extendibility criterion of taxonomies. Fourth, the data set used for taxonomy developments is not comprehensive as only AI use cases from BestPractice.AI were collected.

\section{Concluding remarks}

We extracted the key characteristics necessary for a strategic assessment of an AI use case in terms of its potential business value contribution, and consolidated these in a taxonomy. Our taxonomy provides managers with a guiding tool for informed decision-making when deploying AI in businesses and shall help them to reap the benefits of AI in a structured manner. The taxonomy provides researchers with a better understanding of the impact of AI on businesses in terms of one AI use case's capability of creating various forms of business value in different organizational contexts. This shall pave the way for IS research to enhance theorizing on AI business value in organizations. Launching from this paper, we see future research avenues. For instance, the taxonomy can be adapted and extended to focus on a deeper level of abstraction. In fact, it could take into consideration aspects covering specific resources needed for deployment and running AI use cases, as well as other time-strategic questions such as time required for implementation. Another potential area for future research would be to classify additional AI use cases from sources other than the one we drew from (BestPractice.AI), and combine them with the already collected AI use cases to reaffirm, extend or adapt the taxonomy, and to also engage in cluster analysis. With help of the cluster analysis, archetypes could be identified that would provide insights into the most common structures of business value contributions from AI use cases at the organizational level.

\section{References}

[1] Alsheibani, S., C. Messom, and Y. Cheung, "Re-thinking the Competitive Landscape of Artificial Intelligence", 53rd HICSS, (2020), 5861-5870.

[2] Alsheibani, S., C. Messom, Y. Cheung, and M. Alhosni, "Winning AI Strategy: Six-Steps to Create Value from Artificial Intelligence", AMCIS, (2020), 1-10.

[3] Alsheibani, S.A., D. Cheung, D. Messom, and others, "Factors inhibiting the adoption of artificial intelligence at organizational-level: A preliminary investigation", Twenty-fifth AMCIS, (2019), 1-10.

[4] Amer, M., and T. Maul, "A review of modularization techniques in artificial neural networks", Artificial Intelligence Review 52(1), 2019, pp. 527-561.

[5] Bawack, R.E., S.F. Wamba, and K.D.A. Carillo, “A framework for understanding artificial intelligence research: insights from practice", Journal of Enterprise Information Management, 2021.

[6] Brakemeier, H., P. Gerbert, P. Hartmann, A. Liebl, M. Schamberger, and A. Waldmann, Applying AI: How to find and prioritize AI use cases, 2020.

[7] Bughin, J., E. Hazan, S. Ramaswamy, et al., Artificial Intelligence the Next Digital Frontier?, 2017.

[8] Cheung, C.F., W.B. Lee, and Y. Wang, "A multi-facet taxonomy system with applications in unstructured knowledge management", Journal of knowledge management, 2005.

[9] Coombs, C., D. Hislop, S.K. Taneva, and S. Barnard, "The strategic impacts of Intelligent Automation for knowledge and service work: An interdisciplinary review", JSIS 29(4), 2020, pp. 1-30.

[10] Crews, C., "What Machine Learning Can Learn from Foresight: A Human-Centered Approach", ResearchTechnology Management 62(1), 2019, pp. 30-33.

[11] Davenport, T.H., and R. Ronanki, "Artificial intelligence for the real world", HBR 96(1), 2018, pp. 108-116.

[12] Dellermann, D., A. Calma, N. Lipusch, T. Weber, S. Weigel, and P. Ebel, "The future of human-AI collaboration: a taxonomy of design knowledge for hybrid intelligence systems", Proceedings of the 52nd HICSS, (2019), 274-283.

[13] Dellermann, D., P. Ebel, M. Söllner, and J.M. Leimeister, "Hybrid intelligence", BISE 61(5), 2019, pp. 637-643.

[14] Fadler, M., and C. Legner, "Understanding the Impact of Machine Learning on Enterprise Data Management: A Taxonomic Approach", Pre-ICIS SIGDSA Symposium on Inspiring mindset for Innovation with Business Analytics and Data Science, (2019), 1-3.

[15] Gerbert, P., P. Hartmannn, A. Liebl, and M. Trost, Artificial Intelligence for Boards - Gearing up for the Future of Business, 2020.

[16] Gordon, T., and S. Greenman, "Danske Bank", Johns Hopkins University, 2021. https://www.bestpractice.ai/studies/dankse_bank_identif ies_fraudulent_online_banking_customers_with_99_7_a ccuracy_in_a_pilot_with_the_behaviosec_behavioural_biometrics_system

[17] Grguric, A., E. Vlacic, and N. Drvenkar, "Assessing Firms' Competitiveness and Technological Advancement by Applying Artificial Intelligence as a 
Diffferentiation Strategy - A Proposed Conceptual Model", Economic and Social Development: Book of Proceedings, 2020, pp. 43-61.

[18] Haenlein, M., and A. Kaplan, "A Brief History of Artificial Intelligence: On the Past, Present, and Future of Artificial Intelligence", California Management Review 61(4), 2019, pp. 5-14.

[19] Hartmann, P.M., M. Zaki, N. Feldmann, and A. Neely, "Capturing value from big data - a taxonomy of datadriven business models used by start-up firms", International Journal of Operations \& Production Management 36(10), 2016, pp. 1382-1406.

[20] Hunke, F., C. Engel, R. Schüritz, and P. Ebel, "Understanding the Anatomy of Analytics-Based Services - A Taxonomy to Conceptualize the Use of Data and Analytics in Services", 27th ECIS, (2019), 1-17.

[21] Jain, H., B. Padmanabhan, P.A. Pavlou, and R.T. Santanam, "Special Issue of Information Systems Research-Humans, Algorithms, and Augmented Intelligence: The Future of Work, Organizations, and Society", ISR 29(1), 2018, pp. 250-251.

[22] Jarrahi, M.H., "Artificial intelligence and the future of work: Human-AI symbiosis in organizational decision making", Business Horizons 61(4), 2018, pp. 577-586.

[23] Kohli, R., and V. Grover, "Business value of IT: An essay on expanding research directions to keep up with the times", JAIS 9(1), 2008, pp. 1.

[24] Kukačka, J., V. Golkov, and D. Cremers, "Regularization for deep learning: A taxonomy", arXiv preprint arXiv:1710.10686, 2017.

[25] Longhurst, R., "Semi-structured interviews and focus groups", Key methods in geography 3(2), 2003, pp. 143 156.

[26] Ma, Y., and K.L. Siau, "Artificial intelligence impacts on higher education”, MWAIS Proceedings 42(5), 2018.

[27] Mankins, J.C., "Technology readiness assessments: A retrospective", Acta Astronautica 65(9-10), 2009, pp. 1216-1223.

[28] Manyika, J., M. Chui, B. Brown, et al., Big data: The next frontier for innovation, competition, and productivity, McKinsey Global Institute, 2011.

[29] Melville, N., K. Kraemer, and V. Gurbaxani, "Information technology and organizational performance: An integrative model of IT business value", MIS quarterly, 2004, pp. 283-322.

[30] Mikalef, P., M. Boura, G. Lekakos, and J. Krogstie, "Big data analytics and firm performance: Findings from a mixed-method approach", Journal of Business Research 98, 2019, pp. 261-276.

[31] Mikalef, P., S.O. Fjørtoft, and H.Y. Torvatn, "Developing an Artificial Intelligence Capability: A Theoretical Framework for Business Value", International Conference on Business Information Systems, (2019), 409-416.

[32] Mikalef, P., and M. Gupta, "Artificial intelligence capability: Conceptualization, measurement calibration, and empirical study on its impact on organizational creativity and firm performance", Information \& Management 58(3), 2021, pp. 103434.

[33] Nahm, A.Y., S.S. Rao, L.E. Solis-Galvan, and T.S. RaguNathan, "The Q-sort method: assessing reliability and construct validity of questionnaire items at a pre-testing stage", Journal of Modern Applied Statistical Methods 1(1), 2002, pp. 15.

[34] Nickerson, R.C., U. Varshney, and J. Muntermann, “A method for taxonomy development and its application in information systems", EJIS 22(3), 2013, pp. 336-359.

[35] Olavsrud, T., "5 famous analytics and AI disasters", CIO, 2020. https://www.cio.com/article/3586802/5-famousanalytics-and-ai-disasters.html

[36] Oz, E., "Information technology productivity: in search of a definite observation", Information \& management 42(6), 2005, pp. 789-798.

[37] Pentland, A.P., and M.A. Fischler, "A More Rational View of Logic, Or, Up Against the Wall, Logic Imperialists!", AI Magazine 4(4), 1983, pp. 15.

[38] Porter, M.E., Competitive Advantage, Free Press, New York, 1985.

[39] Porter, M.E., "The value chain and competitive advantage", Understanding business processes 2, 2001, pp. 50-66.

[40] Ransbotham, S., S. Khodabandeh, D. Kiron, F. Candelon, M. Chu, and B. LaFountain, "Expanding AI's impact with organizational learning", MIT Sloan Management Review and Boston Consulting Group, 2020.

[41] Ransbotham, S., D. Kiron, P. Gerbert, and M. Reeves, "Reshaping business with artificial intelligence: Closing the gap between ambition and action", MIT Sloan Management Review 59(1), 2017.

[42] Rao, A.S., and G. Verweij, Sizing the prize: What's the real value of AI for your business and how can you capitalise?, 2017.

[43] Schryen, G., "Revisiting IS business value research: what we already know, what we still need to know, and how we can get there", EJIS 22(2), 2013, pp. 139-169.

[44] Schwarz, A., M. Mehta, N. Johnson, and W.W. Chin, "Understanding frameworks and reviews: a commentary to assist us in moving our field forward by analyzing our past", ACM SIGMIS Database: the DATABASE for Advances in Information Systems 38(3), 2007, pp. 2950 .

[45] Shrestha, Y.R., V. Krishna, and G. von Krogh, "Augmenting organizational decision-making with deep learning algorithms: Principles, promises, and challenges", Journal of Business Research 123, 2021, pp. 588-603.

[46] Wamba-Taguimdje, S.-L., S. Fosso Wamba, J.R. Kala Kamdjoug, and C.E. Tchatchouang Wanko, "Influence of artificial intelligence (AI) on firm performance: the business value of AI-based transformation projects", Business Process Management Journal, 2020.

[47] Webster, J., and R.T. Watson, "Analyzing the Past to Prepare for the Future: Writing a Literature Review", MIS Quarterly 26(2), 2002, pp. 13-23.

[48] Wilson, H.J., and P.R. Daugherty, "Collaborative intelligence: humans and AI are joining forces", Harvard Business Review 96(4), 2018, pp. 114-123.

[49] Yampolskiy, R. V, "Taxonomy of pathways to dangerous artificial intelligence", Workshops at the thirtieth AAAI conference on artificial intelligence, (2016). 